\title{
Improvement of the Automatic Grid Adaptation for Vortex Dominated Flows using Advanced Vortex Indicators with the DLR-Tau Code
}

\author{
M. Widhalm ${ }^{1}$, Andreas Schütte ${ }^{1}$, Thomas Alrutz ${ }^{2}$ and Matthias Orlt ${ }^{2}$ \\ 1 DLR, Institute of Aerodynamics and Flow Technology, \\ Lilienthalplatz 7, D-38108 Braunschweig, Germany \\ 2 DL R, Institute of Aerodynamics and Flow Technology, \\ Bunsenstrasse 10, D-37073 Göttingen, Germany \\ thomas.alrutzedlr.de, matthias.orltedlr.de
}

\begin{abstract}
Summary
Vortex dominated flows appear in many flow simulations such as wake turbulence of an aircraft or a delta wing at a high angle of attack. For detailed investigations of vortex breakdown, vortex interactions or tracing vortex cores, an automated grid adaptation with suitable vortex indicators is essential. Physical indicators, e.g. the vorticity magnitude or the total pressure loss, are in most cases not sufficient for correctly identifying a vortex core.

This paper presents advanced vortex core indicators which properly identify a vortical structure independent of the flow case. These vortex indicators are tested in typical flow applications to determine the right cut-off value which is important for an automated adaptation procedure.

A grid refinement for a delta wing testcase in combination with the newly introduced vortex indicators will demonstrate the improvements compared to the standard pressure loss indicator.
\end{abstract}

\section{Introduction}

Over the past few years physical vortex indicators, e.g. the magnitude of vorticity or total pressure loss were used with the DLR Tau-Code [4] grid adaptation for vortical structure refinements. Recently, vortex identifications based on the kinematics implied by the velocity gradient tensor $\nabla V$ have been proposed in the literature and implemented in the Tau-Code. In addition, the normalized helicity as a vortex indicator has also been introduced. These indicators are local or point-methods where a function can be evaluated grid point by grid point. According to a criterion based on the point values it can classify each point being inside or outside a vortex. For resolving vortex dominated flows, an automated grid adaptation used with flow independent vortex indicators will be an efficient approach. Adapting only the relevant vortical structures is the main issue for many applications and will be presented here for a delta wing testcase. 


\section{Galilean Invariant Indicators}

Chong et al [3] used the critical point theory to describe the topological features of flow patterns by forming a local Taylor series expansion of the flow field. The solution trajectories can be related and classified with three matrix invariants.

In a fluid flow the velocity gradient tensor $\nabla V_{i, j}$, computed from the nondimensionalised velocity vector, can be decomposed into a symmetric $(S)$ and antisymmetric $(\Omega)$ part:

$$
\nabla V_{i, j}=\left(\begin{array}{lll}
\frac{\partial u}{\partial x} & \frac{\partial u}{\partial y} & \frac{\partial u}{\partial z} \\
\frac{\partial v}{\partial x} & \frac{\partial v}{\partial y} & \frac{\partial v}{\partial z} \\
\frac{\partial w}{\partial x} & \frac{\partial w}{\partial y} & \frac{\partial w}{\partial z}
\end{array}\right)=S_{i, j}+\Omega_{i, j}
$$

The symmetric part $S_{i, j}$ is defined by using the index notation with the Einstein summation convention as:

$$
S_{i, j}=\left(\begin{array}{ccc}
\frac{\partial u}{\partial x} & \frac{1}{2}\left(\frac{\partial u}{\partial y}+\frac{\partial v}{\partial x}\right) & \frac{1}{2}\left(\frac{\partial u}{\partial z}+\frac{\partial w}{\partial x}\right) \\
\frac{1}{2}\left(\frac{\partial v}{\partial x}+\frac{\partial u}{\partial y}\right) & \frac{\partial v}{\partial y} & \frac{1}{2}\left(\frac{\partial v}{\partial z}+\frac{\partial w}{\partial y}\right) \\
\frac{1}{2}\left(\frac{\partial w}{\partial x}+\frac{\partial u}{\partial z}\right) & \frac{1}{2}\left(\frac{\partial w}{\partial y}+\frac{\partial v}{\partial z}\right) & \frac{\partial w}{\partial z}
\end{array}\right)
$$

and $\Omega_{i, j}$ is:

$$
\Omega_{i, j}=\left(\begin{array}{ccc}
0 & \frac{1}{2}\left(\frac{\partial u}{\partial y}-\frac{\partial v}{\partial x}\right) & \frac{1}{2}\left(\frac{\partial u}{\partial z}-\frac{\partial w}{\partial x}\right) \\
\frac{1}{2}\left(\frac{\partial v}{\partial x}-\frac{\partial u}{\partial y}\right) & 0 & \frac{1}{2}\left(\frac{\partial v}{\partial z}-\frac{\partial w}{\partial y}\right) \\
\frac{1}{2}\left(\frac{\partial w}{\partial x}-\frac{\partial u}{\partial z}\right) & \frac{1}{2}\left(\frac{\partial w}{\partial y}-\frac{\partial v}{\partial z}\right) & 0
\end{array}\right)
$$

The symmetric Part $S_{i, j}$ is the rate-of-strain tensor and $\Omega_{i, j}$ is the rotation tensor. Computing the eigenvalues of $\nabla V_{i, j}$ in Eq. 1 the following characteristic equation is satisfied:

$$
\lambda^{3}+P \lambda^{2}+Q \lambda+R=0
$$

$\mathrm{P}, \mathrm{Q}$ and $\mathrm{R}$ are the three Galilean invariants and read as

$$
P \equiv u_{i, i}, Q \equiv \frac{1}{2}\left(u_{i, i}^{2}-u_{i, j} u_{j, i}\right), R \equiv \operatorname{det}\left(u_{i, j}\right) .
$$

Second Invariant $\mathbf{Q}$ Hunt et al. [5] defined a vortex as the region with a positive second invariant, $Q>0$. The second invariant is derived from the characteristic Eq. 4 and is defined as written in Eq. 5.

Kinematic Vorticity Number $N_{k}$ Truesdell [12] defined the kinematic vorticity number to measure "the quality of rotation". He defined $N_{k}$ as:

$$
N_{k}=\frac{\|\Omega\|}{\|S\|}
$$


Melander and Hussein's [8] investigations of vortex core dynamics identified the core as a region with $N_{k}>1 . N_{k}$ is non-dimensionalized by the magnitude of strain rate and identifies vortices with large and small vorticity as long as the quality of rotation is the same for both.

$\lambda_{2}$ Criterion Jeong and Hussain [6] introduced a definition of a vortex in terms of the eigenvalues of the symmetric tensor $S^{2}+\Omega^{2}$. The vortex core is defined as a region where two negative eigenvalues of $S^{2}+\Omega^{2}$ appear. Note, that $S^{2}+\Omega^{2}$ is symmetric and the eigenvalues are real values. The vortex core is identified with the requirement:

$$
\lambda_{1} \leq \lambda_{2} \leq \lambda_{3} \quad \text { and } \lambda_{2}<0
$$

\section{Normalized Helicity $\boldsymbol{H}_{n}$ - Stream Vorticity}

In comparison to the indicators above Levy, Degani and Seginer [7] introduced the normalized helicity. Two major shortcomings of the above indicators are the inability of indicating the swirl direction of the vortex and they are unable to differentiate between primary and secondary vortices. To locate and identify coherent structures the normalized helicity is used:

$$
H_{n}=\frac{\vec{v} \cdot \operatorname{rot} \vec{v}}{|\vec{v}| \cdot|\operatorname{rot} \vec{v}|}=\cos \alpha .
$$

High values of helicity reflect regions with high velocity and vorticity whenever both vectors get close to parallel. The cosine between the two vectors provides a sign and shows the direction of swirl in relation to the streamwise velocity field. $H_{n}$ differentiates between primary and secondary vortices because it is directly related to the velocity vector. $H_{n}$ was succesfully used for a delta wing at a high angle of attack by Alrutz and Rütten [1].

\section{Cut-off Analysis for the Advanced Indicators}

Cut-off values control the representation of the vortex core by iso-surfaces. Each grid cell inside the iso-surface will be refined by the adaptation procedure. Due to their invariant behavior these thresholds almost remain constant for a wide range of applications. But, as mentioned by Miliou [10], some indicators, e.g $\lambda_{2}$ criterion, are very sensitive.

Figure 1, 2 and 3 show the $\lambda_{2}$ criterion for a low aspect ratio wing with RAE 2822 airfoil sections. Figure 1 indicates the $\lambda_{2}$ iso-surfaces very close to the theoretical value of zero. Far behind the wing many grid cells are visible with a very small vortex activity and they are not important for the main vortex structure at the wing's side edges. Refining all these grid cells would add points not required for vortex identification. Figure 2 shows the $\lambda_{2}$ iso-surface at a cut-off value of 0.001 . Most of the grid cells far behind the wing are not considered anymore. Some grid cells are still marked where the vortex core is weak. Figure 3 shows a stable vortex core on each side of the wing. 


\section{The Automated Flow and Grid Adaptation Chain}

\subsection{Gathering experience with different vortex indicators}

The EC 145 is a midsize two engine helicopter. Experimental measurements have shown a separation at the back door below the tail rotor boom under certain flow conditions. Subsequently, a flow simulation was carried out to investigate the separation behind the helicopters fuselage in more detail. The computation was performed with a Mach number of 0.2081 and a Reynolds number of 4.33 million. The angle of attack is zero degrees. The main and rear rotor were modeled by actuator discs. In Figure 4-9 the iso-surfaces of the indicators at different cut-off values are shown. The vorticity magnitude $|\omega|$ and the total pressure $P_{\text {tot }}$ values are found through inspection of visualized iso-surfaces. The invariant indicators and $H_{n}$ had to be changed slightly from their theoretical value and in these cases the iso-surfaces indicate the region of the grid which will be refined. The figures are shaded with the first component of the vorticity vector to detect the rotation direction, except the normalized helicity $H_{n}$ which provides the sign of swirl directly.

All the indicators were able to detect the separation at the tail rotor boom. The normalized helicity $H_{n}$ was able to split the swirl action from the rotor discs. Q, $N_{k}$ and the $|\omega|$ detected vortices coming from the rotor discs. All approaches except $H_{n}$ erroneously detect vortex structures on the fuselage. Finally, only $H_{n}$ identifies the important sections at the rear fuselage for an efficient adaptation of the separation.

\subsection{Use of vortex indicators in the Tau adaptation}

Tau adaptation uses an edge based local refinement strategy. The main steps are the indication of edges to be divided considering a current solution and the subdivision of all elements which are needed to get a valid grid with respect to the indicated edge subdivisions [2]. Usually the edge indication uses differences of solution values, e. g. $P_{t o t}$ or $|\omega|$, or differences of gradients as sensors in order to minimize these differences which are supposed to be large in regions with large local errors.

Under some circumstances the differences of physical variables produced by the flow solver in the region of vortices are not large enough to resolve these flow phenomena. A possible explanation could be, that the initial grid resolution is too coarse for the flow solver to simulate the vortices sufficiently. So a flattened solution prevents the edge indication step from refining some vortex regions. Some simulations with pre-refined grids [11] seem to back this observation.

On the one side it may be hard to produce pre-refined initial grids for an unknown flow and on the other side it will be very expensive in terms of computational costs to make pre-refinements in all regions which are possible to contain unresolved vortices. Additionally, this method contradicts the idea of an automated grid adaptation. So the use of vortex indicators could be an alternative approach.

The vortex indication of Tau adaptation uses one of the sensors described above for the edge indication. All edges with a point which is found to be in a vortex region are considered. The needed target point number is found by scaling these edges with a power of its length and marking all edges with an indicator larger than a limit. 


\subsection{Adaptation results for a $65^{\circ}$ delta wing with rounded leading edges}

The flow field around a delta wing is well suited as a typical aerodynamic application for vortex dominated flows. The initial grid of the delta wing has a size of 1.8 million grid points. The flow conditions are a Mach number of 0.4 , a Reynolds number of 3 million and an angle of attack of 13 degrees. The indicators are computed with the velocity vector $\mathrm{V}$ which is nondimensionalised by $\left(M * \sqrt{\gamma * p_{r} / \rho_{r}}\right)$ where $\mathrm{M}$ is the Mach number, $p_{r}$ and $\rho_{r}$ are the reference pressure and density.

In this case the flow topology is different to a sharp leading edge case where two primary vortices are formed right from the apex at the leading edge. In a rounded leading edge case two primary vortices on each side of the wing rotate in the same direction, an inner weaker and a stronger outer vortex. The formation of the inner vortex first occurs close to the apex. The stronger outer vortex is formed further downstream. Without going into more detail of the flow physics, this case is well suited to estimate the ability to accurately resolve different kinds of vortex structures within the flow field.

The impact of the grid adaptation is seen after several adaptation steps in Figure 10 left by using the total pressure $P_{t o t}$ and in comparison in Figure 10 right by using the normalized helicity $H_{n}$. The final grid using $P_{t o t}$ contains about 10 million and for $H_{n}$ about 5 million grid points. The resulting grids are compared in a cut plane at 80 per cent of cord length. It is first of all seen that the main flow features (i.e. the vortices) are correctly predicted by the solver and detected by both adaptation indicators, as seen in Figure 10 for the left and right cut plane. It is evident that more flow structures are detected by the $H_{n}$ indicator and these flow structures are more concentrated in the vortex region than in the case of using $P_{t o t}$. One can see that in the case of $H_{n}$ ( right Figure 10) in addition to the inner and outer vortex as well the horse-shoe vortex generated by the strut is well detected in contrast to the $P_{t o t}$ case while introducing only the half number of grid points during the adaptation procedure. In the case of $P_{t o t}$ adaptation the addition of new grid points is concentrated on the strong outer vortex where the total pressure gradients are high. In case of $H_{n}$ adaptation the addition of new grid points is more balanced and weak vortex structures will also be detected and refined.

In Figure 11 the pressure distribution of both CFD solutions in comparison to experimental data obtained by PSP (Pressure Sensitive Paint) measurements is shown. The center and right figure show the solutions of $P_{t o t}$ and the normalized helicity $H_{n}$ adaptation. As discussed before, both numerical solutions are predicting the main flow features correctly compared to the experiment. In both solutions the inner vortex is predicted too weakly. However in the case of $H_{n}$ adaptation the inner vortex is better refined as discussed before. In both cases the outer vortex is predicted to be too strong and appearing too far upstream.

This over-prediction of the outer vertex is mainly dependant on the two equation turbulence models used. Each of the available models have many different modifications or corrections implemented to make allowance for different physical phenomena. An improvement might be a higher order turbulence model solving this vortex dominated flow. 


\section{Conclusion}

The advanced indicators with different cut-off values were presented to accurately identify vortex cores. From the proposed indicators the normalized helicity $H_{n}$ is a very powerful and reliable indicator for complex flows. The Galilean invariant indicators $Q, N_{k}$ and $\lambda_{2}$ are able to identify vortex cores but they tend to identify strong shear flows as well and finding the appropriate cut-off value becomes more sensitive. Thus it appears that the applicable cut-off values need further investigations for each indicator.

A very effective and efficient way is demonstrated for vortex core refinement on the delta wing. The advantage of the specific vortex core refinement is essential and was the proposed aim for implementing the mentioned indicators. Another feature with Galilean invariant and $H_{n}$ in comparison to physical indicators is the refinement of the vortical structure only. Nevertheless, it seems practicable that both physical and advanced indicators are brought together in the grid adaption as sensors for refinement.

\section{References}

[1] Alrutz T., Rütten M.: Investigation of Vortex-Breakdown over a Pitching Delta Wing applying the DLR TAU-Code with Full Automatic Grid Adaptation. 35th AIAA Fluid Dynamics, 6-9 June, Toronto, 2005.

[2] Alrutz T., Orlt M.: Parallel dynamic grid refinement for industrial applications. In Proceedings of ECCOMAS 2006, Egmond aan Zee, The Netherlands, September $5-8$

[3] Chong M.S., Perry A.E., Cantwell B.J.: A general classification of threedimensional flow fields. Phys. Fluids, A 2, 765, 1990.

[4] Gerhold T., Friedrichs O., Evans J., Galle M.: Calculation of Complex threedimensional configurations employing the DLR TAU. AIAA-97-0167, 1997

[5] Hunt J.C.R, Wray A.A., Moin P.: Eddies, stream and convergent zones in turbulent flows. Center for Turbulent Research Report CTR-S88, p. 318, 1988.

[6] Jeong J., Hussain F.: On the identification of a vortex. J. Fluid Mech., pp. 69-94, 1994.

[7] Levy Y., Degani D., Seginer A.: Graphical Visualization of Vortical Flows by Means of Helicity. AIAA Journal, Vol. 28, No. 8, 1990.

[8] Melander M.V., Hussain F.: Polarized vorticity dynamics on a vortex column. Phys. Fluids, A 5, 1992, 1993.

[9] Metcalfe R., Hussain F., Menon S.,Hayakawa M.: Coherent structures in a turbulent mixing layer. Springer, A 5, 1985.

[10] Miliou A., Mortazavi I., Sherwin S.: Cut-off analysis of coherent vortical structure identification in a three-dimesnional external flow. Comptes Rendus Mecanique, pp. 211-217, 2005.

[11] Schütte, A.; Einarsson, G.; Schöning, B.; Raichle, A.; Mönnich, W., Neumann, J.; Arnold, J.; Alrutz, T.: Prediction of the Unsteady Behavior of Maneuvering Aircraft by CFD Aerodynamic, Flight-Mechanic and Aeroelastic Coupling. RTO AVTSymposium Budapest, April 2005.

[12] Truesdell C.: The kinematics of vorticity. Indiana University 1953. 

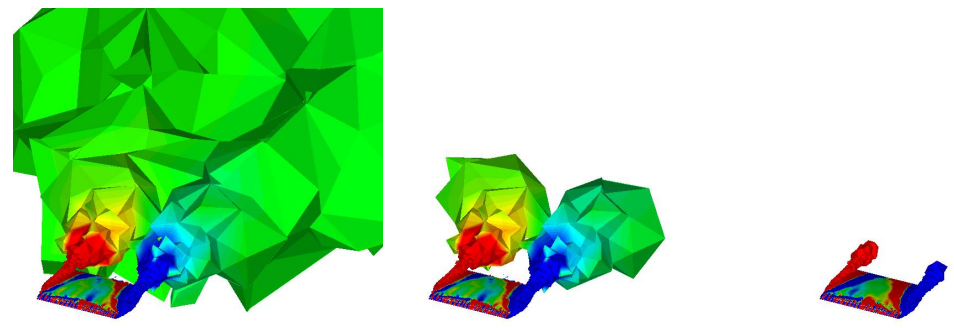

Figure 1 Iso-surface with Figure 2 Iso-surface with Figure 3 Iso-surface with $\lambda_{2}$ values $\leq 0$ $\lambda_{2}$ values $\leq-0.001$

$\lambda_{2}$ values $\leq-1.0$

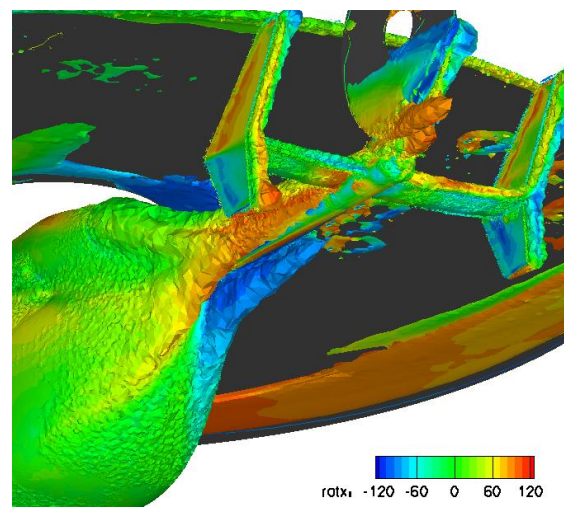

Figure 4 Iso-surface of $|\omega|=100$

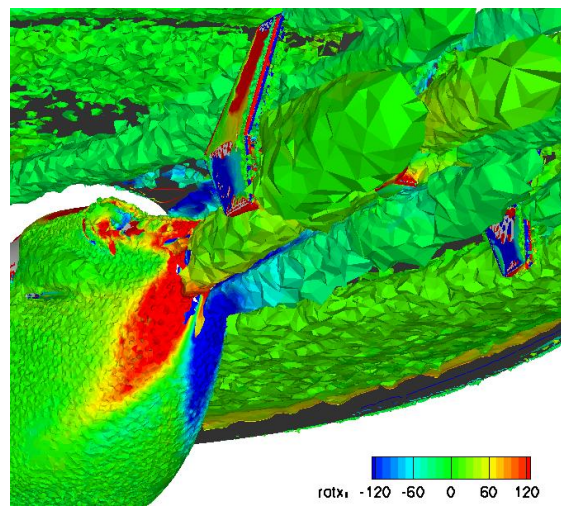

Figure 6 Iso-surface of $Q=0.1$

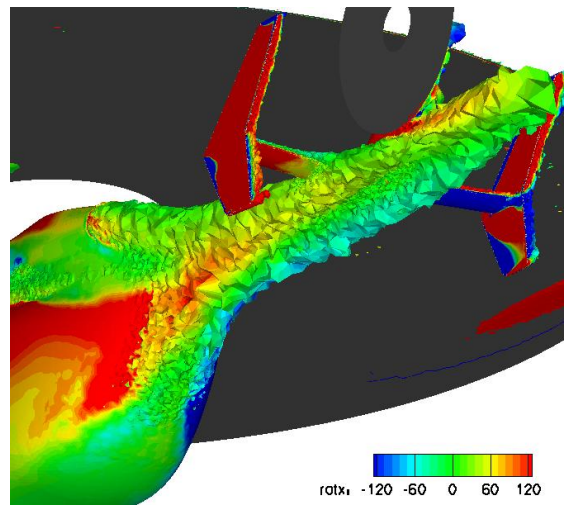

Figure 5 Iso-surface of $P_{t o t}=89.000$

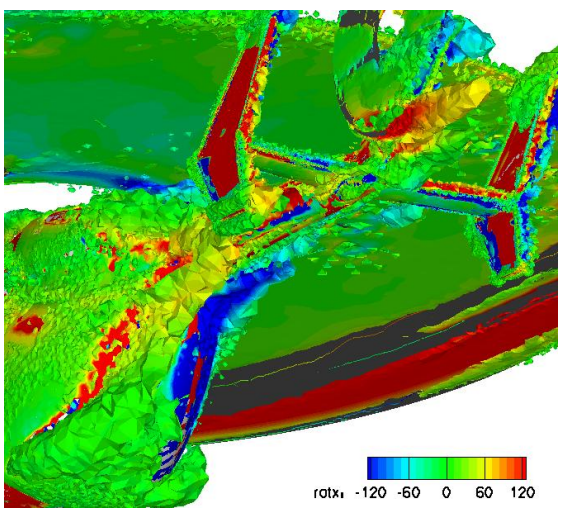

Figure 7 Iso-surface of $\lambda_{2}=-1.0$ 


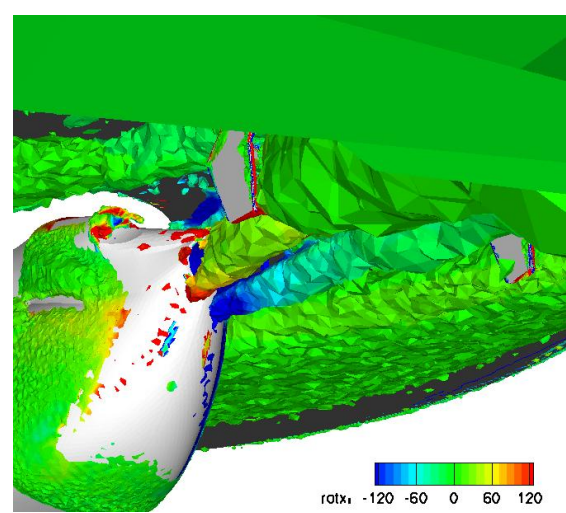

Figure 8 Iso-surface of $N_{k}=1.1$
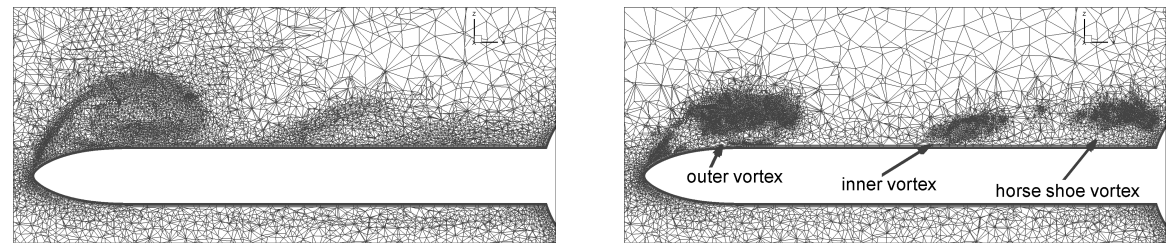

Figure 10 Adapted grid with Ptot (left) and $H_{n}$ (right) at 80 per cent cord length.
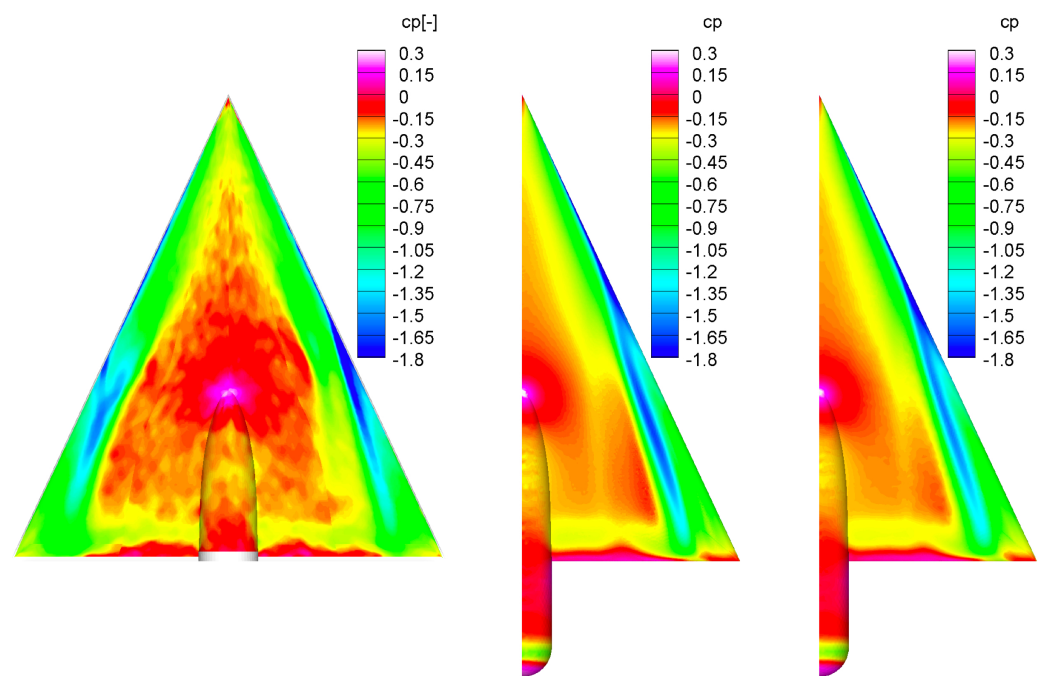

Figure 11 Pressure distribution on the delta wing surface measured by PSP (left) and flow computation after several adaptation steps with the $P_{\text {tot }}$ (middle) and $H_{n}$ indicator (right). 